\title{
UN PLEITO RECURRENTE: \\ EL MONASTERIO DE ARMENTEIRA CONTRA LOS CLÉRIGOS DE LA IGLESIA DE SAN GIAO DO VAL DE MARÍN (1320-1389)*
}

\author{
MIGUEL ROMANÍ MARTÍNEZ** \\ PABLO S. OTERO PIÑEYRO MASEDA***
}

\begin{abstract}
Resumen
La edición de documentos históricos tiene sentido tanto dentro de una colección amplia, como en trabajos aislados que tengan como fin la interpretación de unos determinados hechos del pasado. Este último es aquí el propósito buscado: publicar tres pergaminos inéditos del monasterio de Armenteira pertenecientes al fondo de Oseira, analizando su contenido, su forma y su escritura, junto con índices onomástico y toponímico, y bibliografía.
\end{abstract}

\section{Palabras Clave}

Paleografía, Diplomática, Monasterio.

\begin{abstract}
The edition of historical documents makes sense -in a big collection as well as specific worksto interpretate facts of the past. The aim of this work is to present three unpublished parchments of the Oseira Collection from the Monastery of Armenteira. We analyze their content, form and writing, besides the onomastic and toponimic indices, and an specialized bibliography.
\end{abstract}

\section{Key Words}

Paleography, Diplomatics, Monastery

* Este trabajo se encuadra en el Proyecto financiado por la Xunta de Galicia PGIDT00PX121004.

** Profesor Titular de CC y TT HH de la Universidad de Santiago.

*** Becario Predoctoral del CSIC, programa I3P.- IEGPS CSIC-XdG; participante en el proyecto Diccionario biográfico de la Galicia de los Trastámara (1369-1480) financiado por la Fundación Pedro Barrié de la Maza, dirigido por el Dr. Eduardo Pardo de Guevara. 
$\mathrm{E}$ 1 objeto del presente trabajo es dar a conocer tres documentos en pergamino del monasterio pontevedrés de Armenteira ${ }^{1}$ pertenecientes al fondo del monasterio de Oseira. El contenido de dichos documentos se refiere a los pleitos que, a lo largo del S. XIV, mantiene el monasterio de Armenteira, propietario de la mitad del patronato de la iglesia de San Giao do Val de Marin -hoy llamada San Xián de Marín-, con los clérigos que llevan dicha mitad; la causa de los litigios radica en las rentas que los clérigos deben pagar al monasterio pontevedrés.

La razón de que los tres pergaminos se hallen en el fondo de Oseira, cabe atribuirla al hecho de que este monasterio tenga ya desde el S. XII una granja y diversas propiedades en Marín ${ }^{2}$; en efecto, esta circunstancia motiva sin duda alguna que el granjero de Marín intervenga como mediador en la disputa contenida en el primer documento del apéndice documental; por otra parte se sabe que Oseira tenía el derecho de presentación de la iglesia de San Xián de Marín, que compartía con Armenteira y Poio, lo que justifica, entonces, la conservación de los otros dos documentos en el fondo ursariense ${ }^{3}$.

Los dos primeros pergaminos están escritos, respectivamente, por Alfonso Eanes Jacob, notario público jurado del concejo de la villa de Pontevedra por la Iglesia de Santiago, y por Ruy Pérez, notario de la misma localidad; el tercer documento pertenece a la mano de Alfonso Gómez, el cual se define laconicamente como escripvan sin más precisiones sobre su actividad profesional, lo que hace pensar que sería, quizás, un amanuense al servicio de los monjes de Armenteira. Este documento también está datado en Pontevedra.

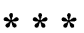

La escritura que exhiben los pergaminos es la habitual del momento en que se realizaron. El primero, cuyo contenido es de 1320, es un traslado notarial hecho en 1446, por ello es el que muestra las caracteríscas gráficas más avanzadas: escritura clara, de buena factura, aspecto redondeado, ausencia de dobles $f, s, r$ y astiles poco elevados; alternancia de $b$ recta y cerrada; predominio de $d$ cerrada, alternan-

\footnotetext{
${ }^{1}$ Véase bibliografía sumaria sobre este monasterio al final de este trabajo.

${ }^{2}$ En 1151, mayo, 12, en Toledo, el rey Alfonso VII dona al abad García y al monasterio de Oseira la villa de Marín en tierra de Morrazo, acotándola jurisdiccionalmente a favor del monasterio; v. doc. $\mathrm{n}^{\circ} 21$ de ROMANÍ MARTÍNEZ, M., "Colección diplomática do mosteiro cisterciense de Santa María de Oseira (Ourense) 1025-1310, Vol. I, Santiago, 1989.

${ }^{3}$ VÁZQUEZ ROUCO, S. "San Xoán de Poio monasterio, coto, parroquia", Diputación Provincial de Pontevedra, Cambados, 1998, p. 110, nota 230.
} 
do con uncial; $h$ recta que alterna con cerrada. Con respecto a los caídos, son rectos en $f, i$ larga y $r$, y cerrados en $s$ larga y $p$, aúnque ésta presente algún ejemplar de caído recto; el caído de $g$ se curva a izquierda, mientras que $h$ e $y$ se proyectan a izquierda y vuelven en sentido contrario en paralelo a la línea de escritura; alternancia de $s$ redonda -ésta especialmente en fin de palabra-y larga; presencia de $v$ y $z$ larga y de $o$ elevada con forma de $v$ en abreviaciones; guiones superfluos, pocas abreviaturas, y envolventes poco numerosos, restringiéndose casi exclusivamente a que. Con respecto al trazado, cabe indicar que presenta contrastes poco acusados. Se trata, pues, de una escritura cortesana, si bien poco desarrollada -no hay $a$ de lineta-, tal como es habitual en Galicia, si se compara con la practicada en la meseta castellana (v. reproducción $\mathrm{n}^{\circ} 1$ ).

La escritura del segundo documento es un buen ejemplo de la letra gótica documental llamada de albalaes, es decir, la típica escritura cursiva documental de este momento en la corona castellana. Se trata de una escritura tendente a la vertical, de perfiles y gruesos poco acusados y cierto grado de angulosidad, sobre todo en la parte central de la escritura, caracterizada por ir las letras de una misma palabra muy pegadas entre sí y por un claro contraste entre el pequeño cuerpo de las letras en la parte central de la escritura y sus astiles y caídos muy desarrollados, especialmente en $s$ y $f$ : a este respecto debe destacarse sobre todo la duplicación de los trazos de estas letras. Otros detalles que pueden señalarse son la alternancia de $s$ redonda con $s$ recta, así como la utilización de la $z$ con forma de 5 y la introducción de la $v$. Es destacable el desarrollo del bucle que abrevia $r$. Finalmente, puede indicarse el comienzo de rasgos volteados en la abreviación de que y en $y$ (v. reproducción $\mathrm{n}^{\mathrm{o}} 2$ ).

El tercer documento datado en 1389, representa un estadio intermedio entre los dos anteriormente descritos. Todavía se conservan los alzados y los caídos bastante prolongados, continuando el tamaño pequeño de las letras en la parte central de la escritura; sin embargo, ya han desaparecido los duplicados en las letras $s$ y $f, y$ hay tendencia al redondeo de los trazos, si bien en general no se aprecian volteos, salvo en la abreviación de algunos que. Asímismo, hay alternancia entre la letra $d$ uncial y la redonda y entre la $s$ redonda -sobre todo en fin de palabra- y la recta. En suma, por las razones expuestas, la escritura de este documento puede clasificarse como precortesana (v. reproducción $\mathrm{n}^{\circ} 3$ ).

Filológicamente, es interesante mencionar algunas palabras usadas en estos documentos, como sajes (doc. $\mathrm{n}^{\mathrm{o}} 1$, aptdo. b) y saies (doc. $\mathrm{n}^{\mathrm{o}} 2$, aptdo. c) para referirse a personas de sabiduría; la expresión di (transcrito $d^{\prime} i$ ) con significado de alli (doc. $\mathrm{n}^{\mathrm{o}} 1$ aptdos. a y b), y el topónimo Logriçaa $\left(\right.$ doc. $\left.\mathrm{n}^{\mathrm{o}} 1\right)$ para referirse al actual Lourizán.

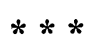


La conservación de los documentos utilizados en este trabajo no es demasiado buena, especialmente en el primero, cuyas líneas iniciales están bastante afectadas, así como su parte izquierda, muy perjudicada por una rotura vertical al sentido de la escritura, que recorre todo el documento de arriba abajo. En general, el pergamino presenta manchones y roturas irregularmente esparcidas por todo el texto, y también diversas partes desvaídas.

Las últimas siete líneas, que contienen las cláusulas del traslado, se escriben con tinta más clara y ello, unido a los importantes desperfectos que presenta esta parte, impiden una lectura completa de los testigos y personas intervinientes en el hecho documentado.

La redacción del documento es bastante enrevesada, según los usos de la época; esta circunstancia y la mala conservación del mismo obligan a que el análisis de su contenido haya de hacerse con mucha atención. Por esta causa, y a efectos de facilitar la comprensión de éste y de los otros dos documentos, en las líneas que siguen trataremos de exponer su contenido en la forma más breve y clara posible.

Documento $n^{\circ}$ 1.- Comienza con una notificación genérica, tras la cual se hace una breve introducción que pone en antecedentes al lector sobre el conflicto - contendas - que había entre el abad de Armenteira y el clérigo de San Xián; a efectos de evitar molestias y gastos, las partes implicadas deciden lo siguiente:

a). -3 de noviembre de 1320 .

Fray Domingo, abad de Armenteira, pedía a Fernando Suárez, clérigo de la mitad de la iglesia de San Xián de Marín, una colleita y un moyo de pan por razón de los derechos ${ }^{4}$ que el monasterio tenía sobre dicha mitad. Para solucionar este problema, el abad y el cillerero de Armenteira, de una parte, y el clérigo Fernando Suárez, de la otra, proponen como jueces árbitros para solucionar el conflicto a doña María, viuda de Fernando Eanes de Meira, y a fray Juan, granjero del monasterio de Oseira, en Marín. El documento está validado por Domingo Eanes, clérigo, notario de Pontevedra y del arcedianato del Salnés, y por Juan de Córdoba, notario de Pontevedra en lugar de Bernald Eanes.

b).- El mismo día, a plazo fijado, las partes fueron convocadas por los jueces designados para que compareciesen a probar et diser sobre esto o que quisesen.

${ }^{4}$ El monasterio de Armenteira tenía la mitad del patronato y derecho a una colleita y un moyo de pan en la iglesia de San Xián de Marín por haberla recibido de Aras Fernández de Meira (v. doc. $n^{\circ}$ 1 aptdo. b y doc. $\mathrm{n}^{\mathrm{o}} 3$ ), hijo de Fernán Eanes de Meira -muerto en el momento en que se produce este acuerdo- $y$ de doña María, la cual interviene en el arbitrio que se describe. 
Efectuada la prueba, los jueces les preguntaron se querian dizer ou rasoar mais, et eles diseron que non. Para concluir, los jueces dictaminaron que el monasterio de Armenteira lleve cada año para siempre una colleita y un moyo de pan por razón de la mitad que el clérigo Fernando Suárez tiene en San Xián de Marín, y que así hagan los otros clérigos que después de este se sucedieren en dicha iglesia; asímismo, los jueces eximen a Fernando Suárez del pago de las colectas no satisfechas a Armenteira hasta el momento, y de costas al monasterio. El documento se otorga en el cabildo del monasterio de San Francisco de Pontevedra, y entre los testigos, destacan Alvar Pérez, hijo de Pedro Álvarez de Sotomayor; Fernando Gutiérrez de Salceda, cavaleiro, y fray Fernando Martínez, fraile de San Francisco. Suscriben, como en el documento anterior, Juan de Córdoba, notario de Pontevedra, y Domingo Eanes.

Documento $n^{\circ}$ 2.- Comienza, también, con una notificación genérica aludiendo al abad del monasterio de Armenteira y a los clérigos de San Xián de Marín, originándose los hechos documentados a) y b) que siguen:

a).- Pontevedra, 22 de enero de 1362.

Esta vez el pleito se resolverá interviniendo el arzobispo de Santiago don Gómez, el cual mediante una carta comisiona como juez al vecino de Pontevedra Juan Núñez da Ponte que soubese a verdade deste preito...

En este momento, la mitad de la iglesia de San Xián de Marín es llevada por dos clérigos: Gonzalo Pérez, canónigo de Santiago en calidad de clérigo beneficiado simple, y por Alfonso Martín, clérigo con cura y también beneficiado. Ambos se niegan a pagar al abad don Fernando de Armenteira la colleita argumentando que el abad y monasterio no tienen derecho a ella.

En consecuencia, el arzobispo compostelano emplaza bajo pena de excomunión a las partes implicadas para que en el plazo propuesto declaren ante Juan Núñez da Ponte, el juez comisionado; sin embargo, cumplido el plazo sólo comparece el clérigo Alfonso Martín. El documento no explica la ausencia de Gonzalo Pérez, el canónigo de Santiago.

b).- 29 de enero de 1362, en las casas del monasterio de Armenteira en Pontevedra.

El abad de Armenteira alega que él y su monasterio cobraban cada año por la mitad de la iglesia de San Xián huna colleyta de pam et de vino et de pescado et de çevada como ya habían hecho sus antecesores, recibiéndola en otro tiempo del clérigo Fernando Suárez ${ }^{5}$ y después, del cardenal Nuño Fernández, y al falleci-

\footnotetext{
${ }^{5}$ Véase doc. $\mathrm{n}^{\circ} 1$
} 
miento de éste, la recibió del ya citado Alfonso Martín, el cual ahora maliçiossamente et em perigoo de sua alma se negaba a pagarla, porque, según él alegaba, el monasterio no tenía derecho a la colecta por la mitad de la iglesia.

El abad don Fernando presenta testigos en apoyo de su demanda ante el juez, cuyas declaraciones son publicadas ante el abad y el clérigo, pidiendo éste un plazo para alegar sus derechos.

c). -8 de julio de 1362 .

En el término fijado comparecieron ante el juez el abad don Fernando y el clérigo Alfonso Martín, el cual dijo que con o dito dom abbade non quiria sobre la dita colleyta andar a preito, mais que lle quiria dar a sua parte da dita colleyta, segundo que lla senpre deran seus anteçesores.

El juez, tras las oportunas deliberaciones, sentencia a tenor de las pruebas practicadas, que el clérigo Alfonso Martín debía pagar cada año al abad y a sus sucesores la mitad de la colleita. Asimismo, sentencia que el abad es libre de demandar a Gonzalo Pérez, el canónigo de Santiago, la otra mitad de la colleita que tiene por el quiñón de dicha iglesia.

Suscribe el documento Ruy Pérez, notario de Pontevedra, por mandato del juez comisionado Juan Núñez da Ponte y del abad de Armenteira.

Documento $n^{\circ}$ 3.- Pontevedra, miércoles, 1 de septiembre de 1389.

Veintisiete años después del último pleito y sentencia sobre el derecho de Armenteira a la percepción de la colleita de San Xián de Marín, vuelve a surgir litigio. En esta ocasión, Gonzalo Martínez, chantre de Tuy y vicario general del arcedianato del Salnés será el encargado de juzgar el caso.

Don Alfonso Domíguez, abad de Armenteira, demanda a Martín Anes, clérigo con cura de la cuarta parte de la iglesia de San Xián, el pago de los polémicos derechos, que esta vez consisten en huun moyo de pan por la medida de Pontevedra, et huna colleyta de pan et vinno et mais un pescado, recordando que estos derechos proceden de la donación de la mitad del patronato de dicha iglesia que en su momento hizo Aras Fernández de Meira ${ }^{6}$ al monasterio.

Martín Anes replica que es cierto que el monasterio debe percibir la renta estipulada sobre la mitad de dicha iglesia de la que es patrón el monasterio, pero alega que él sólo lleva la cuarta con cura de la iglesia, y que Gonzalo Pérez Corvacho, chantre de Santiago ${ }^{7}$, lleva la otra cuarta parte sen cura que fora departida da dita

\footnotetext{
${ }^{6}$ Véase doc. $\mathrm{n}^{\mathrm{0}} 1$, apartado $\mathrm{b}$.

${ }^{7}$ A efectos históricos es interesante mencionar que Gonzalo Pérez Corvacho, chantre de Santiago, podría ser hermano de Vasco Pérez Corvacho, citado por ANSELMO LÓPEZ CARREIRA en
} 
meadade con cura da dita iglesia que tevera o dito Fernán Suares ${ }^{8}$; por lo tanto, él no estaba obligado a pagar más que la mitad de los derechos, y el chantre de Santiago la otra mitad.

El juez encargado, Gonzalo Martínez, vistas las alegaciones, vista una sentencia anterior ${ }^{9}$ y vista la confesión de Martín Anes, halló que éste, en efecto, no era obligado a pagar más que la mitad do dito moyo de pan et a meadade da dita colleita, quedando eximido de pagar lo que debía a Armenteira hasta este momento; con respecto al chantre de Santiago, Gonzalo Pérez Corvacho, resolvió que el abad don Alfonso podía demandarlo se vir que lle conprir... pois ten o outro dito quarto da sua apresentaçon.

Interviene como testigo de esta escritura, entre otros, el escudero Ruy de Ouviña. Suscriben la escritura el juez propuesto que firma como cantor tudensis y el escribano Alfonso Gómez.

A modo de conclusión, se puede decir que el hecho de que en un caso intervengan personajes destacados como el arzobispo de Santiago y el chantre de Tuy, vicario general del arcedianato del Salnés, permite suponer que los derechos que estaban en juego no eran irrelevantes para el monasterio de Armenteira; por la misma razón, puede pensarse que si los clérigos se resisten a pagar, es porque esos derechos suponen para éllos un ingreso a tener en cuenta.

Otra razón que abona esta idea, se manifiesta en que dos miembros del cabildo compostelano - un canónigo y un chantre- se interesen en el disfrute de las rentas que se derivan de la parte del beneficio, cuya voz detenta el monasterio de Armenteira.

Con respecto a las sentencias que a lo largo del tiempo se dictan $-\mathrm{y}$ en las que siempre se insiste en que los clérigos deben satisfacer los derechos correspondientes al monasterio de Armenteira-, es evidente que sus disposiciones caen en saco roto cuando los clérigos se niegan a pagar; es decir, el efecto coactivo de estas sentencias no parece muy eficaz. Habría pues que considerar, o bien dejadez por parte de las autoridades monásticas, o períodos de dificultades en los que la auto-

\footnotetext{
"Estudio sincrónico da economía de Ourense no século XV”, Apéndice documental, doc. de 1382, marzo, 30, Memoria de Licentura inédita, Universidad de Santiago.

${ }^{8}$ Se trata del clérigo demandado en el doc. $\mathrm{n}^{\circ} 1$.

${ }^{9}$ Se refiere a la sentencia pronunciada en el doc. $n^{\circ} 1$.
} 
ridad monástica parece mermada ${ }^{10}$. Un indicio de la falta de capacidad resolutiva del monasterio sería la actitud de aquellos que se niegan a pagar, al parecer durante algunos años, y que acceden a un acuerdo sabiendo que se le van a condonar las exacciones atrasadas.

Se constata, además, que las sentencias siempre compelen a los clérigos con cura, es decir, a los que ejercen allí de rectores; pero en lo que se refiere a los beneficiados sin cura, es decir, ausentes, las sentencias parecen desentenderse de ellos, indicando vagamente que el abad de Armenteira es libre de demandarlos.

Finalmente, la donación de la parte del patronato de San Xián do Val de Marín al monasterio de Armenteira parece una reminiscencia de tradiciones muy arraigadas en los siglos precedentes.

\section{APÉNDICE DOCUMENTAL ${ }^{11}$}

1320, noviembre, 3. Pontevedra.

Sentencia en el pleito puesto por el abad Domingo del monasterio de Santa María de Armenteira y su cillerero contra Fernando Suárez, clérigo, llevador de la mitad de la iglesia de San Juyao do Val de Marín, por una colleita y un mollo de pan; las partes se someten al arbitrio de doña María, viuda de Fernando Eanes de Meira, y fray Juan, granjero de Oseira en Marín.

MADRID, $A H N, 1542 / 5$, perg., orig., gallego, letra cortesana, 390x395 mm, deteriorado y roto por el lado derecho. Se trata de un traslado realizado en 1446, junio, 6 en Pontevedra por Afonso Eanes Iacob, notario público del concejo de dicha villa.

[Sa]bean [todos como] [conte]ndas que eran ontre don frey Domingo abbade et o convento do moosteyro de Santa Maria d'Armenteira de [huna] parte, et [Fernan

\footnotetext{
${ }^{10}$ Una acertada y sintética visión de estos momentos puede verse en la "Introducción histórica" a cargo del Prof. Francisco Javier Pérez Rodríguez del Libro Tumbo de Pergamino editado por ROMANÍ MARTÍNEZ, M. y RODRÍGUEZ SUÁREZ, Ma. P., Libro Tumbo de Pergamino. Un códice medieval del monasterio de Oseira, Santiago, Tórculo, 2003.

${ }^{11}$ Los autores agradecen las opinones de la Prof ${ }^{\mathrm{a}}$. $\mathrm{Dr}^{\mathrm{a}} \mathrm{M}^{\mathrm{a}}$ José Portela Silva en la interpretación de algunas frases problemáticas.
} 
Soares...] [...] [clerigo de] / San Juyao, conven [a saber ...] sobre colleyta et huun moyo de pan que o dito abbade et convento deman[davan] por cada anno a o dito Fernan [Soares] na dita [...] iglesia que [...] / moost[eyro] [...] [mea]dade av[...] que foi de Meira, cuja voz ese moosteyro h[a], et sobre [...] custas que [ontre] eles sobre esto recreçeron, et sobre t[odas as] custas que hu[uns et o]utros / çebi[...] [... de] seu [pr]azer conprometeron en nos, dona Maria, moller [que f]oi de Fernan Eanes de Meira, et frey Johanes, freire d'Oseira [...] et reçeberemos, por / seus [...] de graça et ar[...] [...] conteudo en huun conprimiso ende feyto por Domingo Eanes et Johan de Cordova, notarios, do qual o thenor a tal [he]:

a).- [Era de mill et trezentos et çinquoen]ta et oyto / [annos], tres dias de no[venbro. Sabe]an todos que nos, frei Domingo abbade et frei Afonso çelareiro do moosteyro de Santa Maria d'Armenteira, por nos et [por] lo con[vento et] voz [dese dito moosteyro] de huna parte, / et eu Fernan [Soa]res clerigo da meadade da eglesia de San Juyaao do Val de Marin, por min et por meus soçesores da outra parte, sobre demandas et contendas que ontre nos avemos [...] colleyta / et moyo de pan, que nos don abbade et convento do dito moosteyro demandamos a vos Fernan Soares, para cada anno en a meadade que teendes da dita eglesia do Val [de Marin ...] estas que [...] ontre nos re/cleçeron, et sobre todos [juiços et] demandas que huuns outros sobre esto ontre nos avemos et aver podemos ata este presente dia, de noso praser conprometemos [... donna Maria] moller que foi de Fernan Eanes / de Meira, et eu frei Johanne frade d'Oseira, grangeiro de Marin et reçebemos eles por nosos juizes de graça, arvidros, [ami]gaveles conpoedores [... mill maravedis da] moeda del rei don Fernando / pagadeiros a a parte agardante este conprimiso da parte non agardante. Asi que quer que os ditos arvidros anbos en senb[la ...] partes sobre isto julgaren, [...] enprasaren, loaren, arvidraren, / deren et por ben teveren asi [en dia] feriado como [non feriado], estando ou seendo nos partes presentes ou non presentes [et a huna de] nos partes presente et a outra non [guar]dado dereito ou non as / guardando, nos partes o [outorg]amos et avemos por firme en todo, et outorgamos de o conprir et non viir contra elo en ningua guisa por nos nen por outro [...] feseremos que / nos non valla nen posamos hy s[er ...] dos et damoslle poder que nos posan chamar et çitar et dar por pertemaz [aquel] de nos que non quiser dar et conprir [...] julgar tanben sobre la / penna et sobre las custas como sobre la $\mathrm{p}[\ldots . .$.$] , et que posan julgar quantas vezes quiseren ata que todo o pleito por$ eles seja libre et por sentença de[finit]iva. Et nos [...] apelar, nen suplicar, nen querelar / a nehuun sennorio de sennor nen avynça que eles ontre nos den. Et quen contra esto vier, peyte a a outra parte [a dita] penna. Et este conprimiso [...] [jul]garen et mandaren seja firme / et estavele para senpre. 
Ts: frey Fe[rnan] Martines, fraire da Orden de San Françisco et Afonso Eanes, clerigo, sobrino de Çibraao Peres, que foi [... Pedro Eanes], dito Salgeiro, d'i do Vale / et Afonso de Cordova, escripvano.

[Et] eu, Domingo Eanes, clerigo, notario de Pontevedra et en terratorio do arçidiagado de Salnes, jurado, a esto fuy presente con Juan de Cordo[va], notario subescripto, et fezemos escripvir / et confirmar, et meu signal hi ponno que tal he.

Et eu Iohan de Cordova, notario de Pontevedra, jurado en lugar de Bernald Eanes, notario da dita villa, a o [que] foi presente con o dito Domingo Eanes, et / notario sobredito, et fezemos escripvir et [confirmar et hi] meu nome et signal do dito Bernald Eanes ponno que tal he.

b).- Et nos, [...] a rogo [das] ditas partes reçebemos o dito pleito / [...] termio a que viesen ante nos a [prob]ar et diser sobre esto o que quisesen.

Et a ese termio vieron ante nos et demandaron [et di]seron et rasoaron [quanto] quiseron, et soubemos sobre isto / a verdade por qua[ntas] partes o mellor podemos sa[ber]. Et disemoslle se querian dizer ou rasoar mais, et eles diseron que non. Et por lo [...] dito avian, çerrav[an] pleito, et pedian sentença.

Et nos, oydas / as demandas et todalas cousas que estas partes ante nos dizer et rasoar quiseron pois que o pleito foy çerrado et termyo asignado para dar sentença [as partes pre]sentes et demandandoa nos, abido / sobre isto consello con sajes por partiremos as ditas partes de pleito et de custas et de danos, et por los trager a aviinça et concordia, porque achamos por verdade que o dito Aras Fernandes de Meira, / cuja voz o dito moosteyro ha, avia cada anno huna colleyta et huun moyo de pan por la medida dereyta de Pontevedra, en a dita meadade que o dito Fernan Soares ten da dita eglesia de San Juyao / do Vale; julgamos et mandamos conpoemos, aviimos et por ben teemos que o dito moosteyro d'Armenteira aja cada anno para senpre en a dita meadade que o dito Fernan Soares ten da dita eglesia / huna colleyta et huun moyo de pan por la medida de Pontevedra. Et que o dito Fernan Soares et os outros clerigos que depos ele y vieren, que lle den ende cada anno por senpre a dita colleyta et / moyo de pan por la dita medida. Et mandamos que sejan quitas a o dito Fernan Soares todas las colleytas que lle non dou do tenpo traspasado ata aqui. Et mandamos que o dito Fernan Soares / seja quito de todas las custas que o dito abbade et convento sobre isto feseron.

Et por isto mandamos que as ditas partes sejan quitas et anuçuadas (sic) et [...]cadas de todas las demandas / et buscas que hunos outros ontre si avian et aver poderian en qualquer guisa por esta rason ata este presente dia. Et a parte deles questo non conprir et co[...] vier, peyte a a outra parte a dita / penna dos ditos myll maravedis que son conteudos en o dito conprimiso. Et esta [avy]nsa este senpre firme en sua revor. 
Et porque esto seja çerto et non vii[na en dul]ta, rogamos a Domingo Eanes et a Johan / de Cord[ova], notarios de Pontevedra, que fezesen [ende e]sta se[ntença] et posesen en ela seus nomes et seus signaes.

Presentes a esta sentença [...] frey A[fonso], çelareiro, por si et por lo convento / do dito moosteyro, et o dito Fernan Soares, por si et por seus suçesores, et en presença de nos, notarios, et das testigos (sic) subescriptas, diseron [...] sobredito he, et sub a dita / penna.

Isto foy en o cabidoo do moosteyro de San Françisco de Pontevedra, tres dias andados do mes de novenbro, era de mill et trezentos et çinquenta et [oy]to annos.

Disto son testigos (sic) / Alvar Peres, fillo de Pedro Alvares de Soutomayor, Fernan Goterrez de Salzeda, cavaleyro, frey Fernan Martines, fraire da Ordeen [de San Françisco... ]drinno de Çibraao Peres, que foi / clerigo de Marin, et Iohan Domingues et Pedro Anes, dito Salgeiro, d'i do Vale et Afonso de Cordova, $\mathrm{s}[\ldots]$ [...], Domingo Eanes [notario de Pontevedra et en terratorio do ar]çidiagado de Salnes, jurado, / presente fuy con lo dito Johan de Cordova, notario so scripto. Quando [os ditos] arvidros deron a dita sentença et [...] outorgaron [...] o sobredito [...] ditos arvidros o fesemos / scripvir et vimos [o dito] [con]primiso [feyto] por nos, et fesemoslo trasladar fielmente et confirmar, et aqui meu no[me et sign]al que tal ponno que tal he.

Et [eu, Johan de Cordova] notario de Pontevedra, ju/rado en lugar de [Bernal]d Eanes, notario da dita villa, presente foi con o dito Domingo Eanes, notarios [...] os ditos [...] a dita sentença, et quando [...] como sobredito he, et / rogados ditos arvitros, a fezemos s[cripbir ...] et darmos o dito conprimiso feyto por nos et $\mathrm{f}[. .$.$] confirmo,$ et aqui meu [...] dito Bernald Eanes, / pono en testemoyo de verdade que tal he./

Este he o traslado dos sobreditos conprimiso et sentença suso encorporados, os quaes eran scriptos en pergameo de coyro, et signado dos signos [de Domingo Eanes et] / Johan de Cordova, notarios, a qual eu, Afonso Eanes Iacob, notario publico jurado do conçello da vila de Pontevedra por la iglesia de Santiago, aqui $[\ldots]$ / et de [...] oreginal donde foy sacado, et bay çerto et o doy a don frey Iohan, abade do moosteyro de Santa Maria d'Armenteira et [...] que para / elo [...] dou [...] Fernan [...] Christovoo de Briallos, bicario en o arçediagado de Salnes, por don Afonso Lopes de Ball[... arçe]diago do dito [arçediagado de Saln]es.

En a dita vila de Pontevedra, / en viinte et seis dias [do mes de] junio do anno do nasçemento de noso Sennor Ihesu Christo de mill et quatroçentos et quarenta et $\mathrm{se}$ [is annos].

Estando ende presentes [por ts. ...] Gonçalvo Estevees, clerigo, / con [cura] de San [Jullao do Val de Ma]rin, [...] de Montillon, clerigo con cura de Santo Andre de 
Logriçaa, et frey Fernando de Deça, fraire do dito moosteyro de San[ta Maria d'Arm]enteira, et Iohan de Rioo, mora/dor na granja de [... aqui] meu nome et signal ponno en testemoyo ( $\mathrm{sic}$ ) de verdade que tal he.

(SIGNO: en el centro Alfonsus; en torno al mismo Jo·ha·nn·is).

1362, julio, 8. Pontevedra

Sentencia a favor del monasterio de Armenteira y su abad don Fernando en el pleito que de nuevo se suscita contra los clérigos de la parroquia de San Giao do Vale; incluye carta de comisión a favor de Juan Núñez da Ponte como juez en la causa (1362, enero, 22) y relato de las pruebas practicadas por el juez (1362, enero, 29).

MADRID, $A H N, 1548 / 20$, perg., orig., gallego, letra de albalaes, 255x562 mm, desvaído en las primeras líneas y roto en su margen derecho al final.

$\mathrm{S}$ [abeam quantos esta carta de comisson biren como ante min Johan Nunnez] da Ponte, juis de comissoon dado por nome do sennor arçobispo/ de Santiago dom Gomez, scripta em pa[pel] selada de seu seelo et roborado de seu nome posto con sua mao, segundo que [por ela paresçia] / [ontre] dom frey Fernando, abbade do moesteyro de Santa Maria d'Armenteyra et o convento do dito moesteyro da huna parte, et ontre Gonçalvo Peres [coengo] / de Santiago, et Affonsso Martin, clerigos da meadade da eglesia de Sam Giao do Vale da outra; apareçeron ante min, o dito dom abbade, por sy et / en nome do dito convento da huna parte, et o dito Affonsso Martin, clerigo da parte con cura da dita iglesia de Sam Giao do Vale, da outra [parte;] / enton o dito dom abbade presentou ante min por este Aras Peres notario soescripto, a dita carta de comissom dada por a[utoridade] / [da qual] carta o tenor a tal he:

a).- Dom Gomez por la graça de Deus arçobispo de Santiago, capellan mayor del rey et notario mayor del rey de Castela et de Leom, a todos os clerigos et leigos que o abade d'Armenteyra por esta nossa carta chama, a cada huun de vos a quen esta nossa / carta for mostrada, saude.

Sabede que o dito abbade et convento do dito moesteiro d'Armenteyra da huna parte, et Gonçalvo Peres [...] / coengo de Santiago, clerigo beneffiçiado en a igle-

Cuadernos de Estudios Gallegos, Tomo LI, Fascículo 117, Santiago 2004. (Págs. 239 - 262) 
sia de Sam Giao do Vale, et Affonsso Martin, clerigo con cura da dita iglesia, da outra, ham [ontre] / sy contenda et demanda por razon que os ditos abbade et convento am huna colleyta em cada huun anno en a dita iglesia em [aquelles lugares ...] / tam solamente de que os ditos Gonçalvo Peres et Affonsso Martin en a dita iglesia som benefiçiados; et os ditos Gonçalvo Peres et Affonsso [Martin ... dizen] / que os ditos abbade et convento non am y a dita colleyta segundo que elles dizen.

Et nos por quitar as partes de custas et de [pleytos ...] / de consentymento das ditas partes, cometemos a Johan Nunnez da Ponte, vezino de Pontevedra, que soubese a verdade deste preito / et colleita por hu quer que a mellor podese saber; et que o livrase et determinase ontre as ditas partes por sentença como achase por dereyto. / Porque vos dizemos et mandamos em virtude de santa obidiençia et so penna d'escomoyon que quando et cada que vos, o dito dom abbade, / ou outro por el, por esta nosa carta chamar et çitar ata sabado viinte et nove dias deste mes, vaades ante o dito Johan Nunnez / a dizer verdade por juramento dos Santos Avangeos, aquela que souberdes et por que fordes preguntados, se os ditos abbade et convento, / de dereyto ou de husso et de custume, am et deven d'aver a dita colleyta en os ditos quinoos da dita iglesia de Sam Giao do / Vale, de que os ditos Gonçalvo Peres et Affonsso Martin em ela som benefiçiados, et se os ditos Gonçalvo Peres et Affonsso Martin lles deven / a dita colleyta em cada huun anno, segundo que os ditos abbade et convento dizen. Et non façades ende $\mathrm{al}^{12}$, so a dita penna.

$\mathrm{Da} / \mathrm{da}$ em Pontevedra, viinte et dous dias de janeiro, era de mill et quatroçentos annos.

Gomecius archiepiscopus compostellanus. Garçia Peres, / notario, por mandado do arçobispo.

b).- Et a dita carta liuda et presentada, o dito dom abbade fez sua demanda ante min por paravoa a o dito Affonsso / Martin em esta guyssa, que como el et o dito moesteyro de Santa Maria d'Armenteyra et seus anteçesores ouvesem cada anno en a dita / meadade da dita iglesia de Sam Giao do Vale, de que eran clerigos os ditos Gonçalvo Peres et Affonsso Martin, et que fora de Fernan Soa/res, clerigo que fora da dita meadade da dita iglesia, huna colleyta de pam et de vino et de pescado et de çevada, et que esta colleyta que / a deran de cada anno en a dita iglesia a seus anteçesores, et a el depoys que foy abbade do dito moesteyro / o dito Fernan Soares; et depoys da morte do dito Fernan Soares, o cardeal Nuno Fernandes,

${ }^{12}$ Lo demás, lo necesario, a mayores. 
que foy clerigo da dita meadade da / dita iglesia. Et depoys da morte do dito Cardeal, que la dou o dito Affonsso Martin en a dita iglesia et se aviinna con el por la / dita colleyta; et estava el et o dito moesteyro et seus anteçesores em jur et posse de aver et levar a dita colleyta de cada anno / dez, vinte et trinta et quoreenta annos a aca. Et que agora este Affonsso Martin maliçiossamente et em perigoo de sua alma, ge la enbar/gava et lla non quiria pagar.

Pedia a min juis, que julgando por sentença lle mandase pagar et dar de cada anno, en a dita mea/dade da dita iglesia a dita colleyta. Contra a qual demanda o dito Affonsso Martin disso que lle negava que o dito moesteyro non ouvera nen a/vya a dita colleita en a dita meadade da dita iglesia; et o dito dom abbade diso que o quiria provar, et pedio a min que lle re/çebese as ts. que sobre esto quissese dar.

Et eu, juis sobredito, reçebi as testemoyas (sic) que o dito dom abbade dou et presentou, et / ajuramenteyas a os Santos Avangeos em pessoa do dito Affonsso Martin, et por lo dito juramento as preguntey et fige preguntar a o dito / notario por lo dito rogo. Et as ditas ts. chamadas, fige ante min viir o dito delas, et figeas publicar em pesoa dos ditos dom / abbade et Affonsso [Martin]; et elas publicadas, o dito Affonsso Martin pid $<i>0$ o traslado delas et termyno para diser contra elas do seu dereyto. Et eu / mandeyllo dar et asyneylle termino para luns primeyro que ven que seeran oyto dias de juyo, a que dissesse do seu dereyto.

Ysto foy / em Pontevedra, en as cassas do dito moesteyro d'Armenteyra, viinte et nove dias de janeyro, era de mill et quatroçentos annos.

Ts. / [...] Pardo, Fernan Anes Mourinno, Gonçalvo Peres de Penente, Nuno Vidal, Fernan, carniçeyro, homes do dito Johan Nunes.

c).- Et depoys / desto, oyto dias do dito mes de juyo da dita era, o dito dom abbade da huna parte, et o dito Affonsso Martin da outra, apareçeron / ante min, et enton o dito Affonsso Martin disso que con o dito dom abbade non quiria (sic) sobre la dita colleyta andar a preito, mays que lle / quiria (sic) dar a sua parte da dita colleyta segundo que lla senpre deran seus anteçesores. Et sobre esto anbas as ditas partes / concludiron et pediron a min juis, que livrase o que achase por dereyto. Et eu, juis sobredito, vista a demanda et petiçon do / dito dom abbade, et a resposta et deffenssom do dito Affonsso Martin, et vista a dita prova que sobre esto foy feyta et / quanto ante min quiseron diser et rasoar, poys que o pleito foy sarrado et dia assynado para dar sentença, as ditas partes / presentes, sentença demandantes, seendo en lugar de julgar et avudo sobre $<\mathrm{e}>$ lo consello con saies, em este presente escripto / julgando por sentença por lo poder que eu he por la dita carta, por quanto achey provada a entençon do dito dom abbade et / por la confesom et resposta do 
dito Affonsso Martin, [man]do que o dito Affonsso Martin de et page de cada anno a o dito dom abbade / et (sic) a seus suçesores a meadade da dita colleyta que lle perteeçe de pagar por lo dito quinon que ten da dita iglesia segundo que achey provado por la dita prova que lla senpre deu o dito Fernan Soares et os outros que foran depoys del clerigos / da dita meadade da dita iglesia de que foy clerigo o dito Fernan Soares; et por esta meesma sentença mando que fique / a salvo a o dito dom abbade de demandar por dereyto a o dito Gonçalvo Peres a outra meadade da dita colleyta por lo dito quinon / que ten da dita iglesia; et a o dito Gonçalvo Peres fique a salvo para se defender a dereyto; presentes o dito dom abbade [...], / Affonsso Martin, et outorgaron a dita sentença.

Et que isto seia çerto rogey et mandey a o dito Aras Peres [...] / esta carta de sentença et a desse a o dito dom abade [...] / ditos [...] juis Affonsso [...] escudeyro, et outros. /

Et eu, Ruy Peres, notario de Pontevedra, [a todo] isto que sobredito he con as ditas ts. / presente foy, et escripvi et confirmo a dita sentença, escripvi por mandado do dito Ioan Nunes, et de man/dado do dito dom abbade que ma pediu, et aqui ponno meu nome et sinnal en testemoio (sic) de verdade / acustumado que tal he (SIGNO).

1389, septiembre, 1 , cuarta feria. Pontevedra.

Una vez más surge la cuestión de las colleitas de San Giao do Val de Marín: Gonzalo Martínez, chantre de Tuy y vicario general del arcedianato del Salnés, resuelve que los llevadores de la mitad con cura de dicha parroquia, paguen una colleita y moyo de pan y vino al monasterio de Armenteira.

MADRID, $A H N, 1551 / 2$, perg., orig., gallego, letra precortesana, 305x281 mm.

Sabbeam quantos esta carta de sentença viren como ante min, Gonçalvo Martines?, chantre de Tui et vigario geeral do arçidiagado de Salnes, [conpareceu] en juiso [ante] / min, dom frey Afonsso Domingues, abbade do moesteyro de Santa Maria d'Armenteyra, por si et en nome do dito moesteyro et convento del, da huna parte; et Martinno Anes, / clerigo da quarta parte con cura da iglesia de San Giao do Val en Marin, da outra parte, et como o dito don abbade sua demanda fezo por 
palavra contra o dito Martinno Anes / en esta gisa: disia que como o dito moesteyro aja de aver et levar cada anno en a metade da dita iglesia, de que Fernan Suares foi clerigo, huun moyo de pan por la medida de Pontevedra, / et huna colleyta de pan et vinno et mais un pescado por r[ason] de padroadego por voz de Aras Fernandes, que foy de Meyra. Et o dito Fernan Suares / et os reytores que foron da dita iglesia que teveron a cura dela pagassen a dita $\mathrm{r}$ [enda] cada anno a o dito moesteyro sen contradita ninhuna. Et este dito Martinno Anes, clerigo, aia et / tena a quarta parte da dita iglesia con a cura et reytoria dela, et recusa de [pagar] o dito moyo de pan et colleyta. Por ende, pedia a min que constrengesse / o dito Martinno Eanes, que lle dese et pagase o dito moyo de pan et colleyta de cada anno a el et a o dito moesteyro.

Et o dito Martinno Anes, clerigo, respondeu et diso que era verdade / que o dito moesteyro avia de aver et levar cada anno en a dita meadade da dita iglesia o dito moyo de pan et colleyta por rason de padroadego, sigundo que o dito don abbade / disia et esteveran et estavan en posison de o levar, et da qual meadade o dito Fernan Suares, seu anteçesor, fora clerigo et reitor, et que el non tiinna mays que / huna quarta con cura da dita iglesia, et Gonçalvo Peres Corvacho, chantre de Santiago, en a outra quarta parte sen cura que fora departida da dita meadade con cura da dita / iglesia que tevera o dito Fernan Suares; et por ende, el de dereyto non era thiudo a pagar mais que a meadade do dito moyo de pan et a meadade da dita colleyta; et o dito / chantre de Santiago, que ten a outra dita quarta parte, avia de pagar a outra meadade; et a o al pedido per (sic) lo dito don abbade, non era thiudo.

Et sobresto os ditos dom / abbade et Martinno Anes, clerigo, pediron a min, que vise o dito et alegado ontr'eles en este dito feyto, et o livrase como achase por dereyto et vise que era ben.

Et eu, / chantre et vigario sobredito, vista a demanda et petiçon do dito don abbade, et vista huna sentença dada por dona Maria, moller que foy de Fernan Anes de Meira, / et por frey Johan, monge d'Oseira, granjero de Marin, homes boos, arvidros ontre [don frey] Domingo, abbade que foy a a sazon do dito moesteyro d'Armenteyra, et Fernan Suares, clerigo da / meadade da dita iglesia de San Gyao do Val de Marin, sobre rason do dito moyo de pan et colleyta; os quaes homes boos mandaron que o dito Fernan Suares, clerigo, et os que / fosen clerigos da meadade da dita iglesia desen et pagasen cada anno a o dito convento et abbades del o dito moyo de pan por la medida de Pontevedra, et a dita colleyta cada / cada (sic) anno por rason de padroadigo.

Et outrosi, vista a resposta et confesion do dito Martinno Anes, et en como non he clerigo mays que do quarto con cura da dita iglesia, / et o dito Gonçalvo 
Peres Corvacho, chantre de Santiago, ten et he clerigo do outro quarto con cura da dita iglesia, o qual he da apresentaçon do dito moesteyro, por ende, acho / que o dito Martinno Anes, clerigo con cura da dita iglesia, non he tiudo a pagar mais que a meadade do dito moyo de pan et a meadade da dita colleita cada anno a o / dito moesteyro d'Armenteyra; et mando a o dito Martinno Anes, clerigo, et a os que d'aqui endeante teveren et ouveren a dita quarta con cura da dita iglesia, que den et pagen d'aqui en/deante cada anno a o dito moesteyro d'Armenteyra et abbades del a dita meadade do dito moyo de pan por la medida por que o ata aqui levaron os abbades que foron do dito moesteyro / ou outros en seu nome, et tanben a meadade da dita colleita; et mando a o dito don frei Afonso, abbade, que demande a o dito chantre de Santiago por la outra medade do dito / moyo de pan et colleyta, pois ten o outro dito quarto da sua apresentaçon, se vir que lle conprir; et dou por quito et livre a o dito Martinno Anes de todo o que devia et avia de / dar ata oi este dia a o dito don abbade ou a outros en seu nome do dito pan [et colleyta].

Et se aqui endeante se alguno demanda a o dito Martinno Anes, clerigo, sobre lo dito moyo / de pan et colleyta do tenpo passado, que o dito abbade et moesteyro $\mathrm{d}$ 'Armenteyra que o faça livre et quite, et seendo en lugar de julgar, o julgo et pronunçio asi por minna sentença de/fenetiva en este presente escripto.

Et esta sentença dou a plazer et consentimento dos ditos don abbade et Martinno Anes, clerigo, os quaes outorgaron et consentiron en ela./

Dada en Pontevedra, quarta feira, o primeiro dia do mes de setenbre, anno do naçemento de Nostro Sennor Jhesu Christo de mill et tresentos et oyteenta et nove annos./

Ts.: Martinno Anes clerigo de coro, Rui d'Ouvinna escudeyro, Johan Suares, Iohan Nunes clerigo de Paradela, Martín Barregan pescador, et Afonso Peres escrivan, et outros./

(Autógrafos) Cantor tudensis. Afonsso Gomes, escripvan. 


\section{MUESTRAS PALEOGRÁFICAS*}

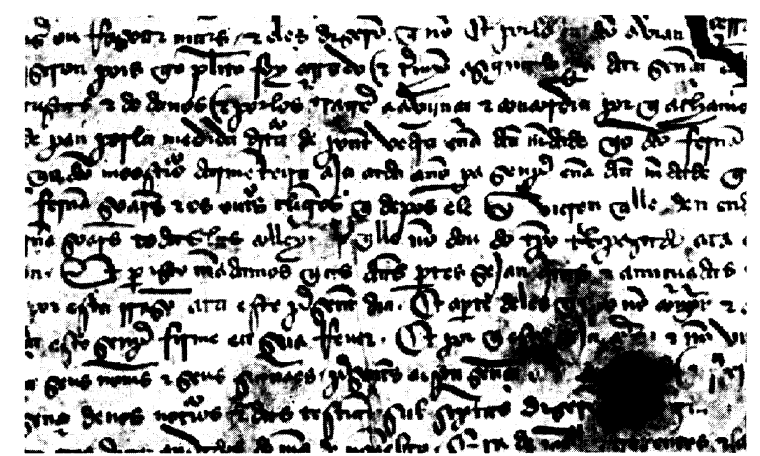

Reproducción 1.- Doc. $n^{\circ} 1,1446$, junio, 6. Escritura cortesana.

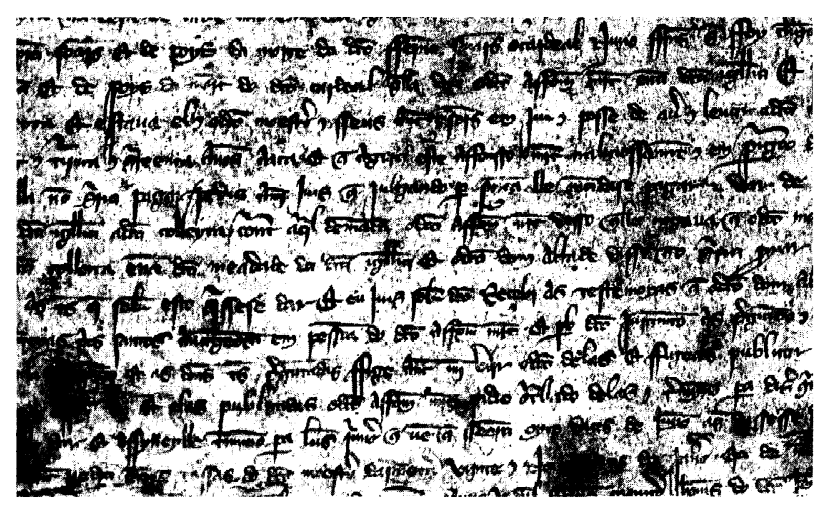

Reproducción 2.- Doc. $n^{\circ} 2,1362$, julio, 8. Escritura de albalaes.

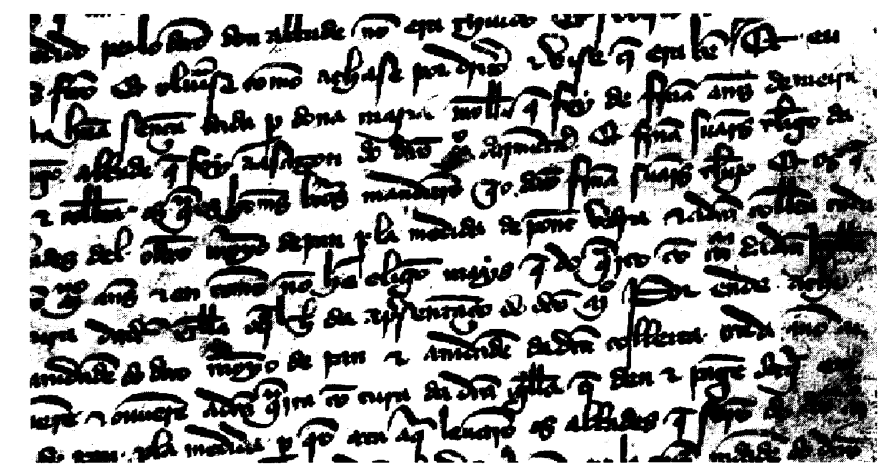

Reproducción 3.- Doc. $n^{\circ} 3,1389$, septiembre, 1. Escritura precortesana.

* Reproducciones digitales: Isam Darwiche Lodeiro (IEGPS-CSIC-XdG). 


\section{ÍNDICE ONOMÁSTICO}

Se encuentran referenciadas todas las personas que se mencionan en la documentación.

El número o números que siguen a cada nombre indican el documento o documentos en el que se encuentra.

El nombre de una misma persona escrito en diferentes grafías en los documentos donde aparece se reduce a su forma más habitual, respetando su ortografía.

El cargo, profesión o filiación que posee una persona se escribe con la grafía actual, para ofrecer mayor claridad y accesibilidad, excepto en aquellas palabras que sólo en su forma arcaica conservan su total significación, como por ejemplo «home».

Los nombres escritos entre paréntesis mencionan el utilizado en la historiografía, para su mejor localización: «[Fernando IV]».

[...] de Montillon, clérigo de San Andrés de Lourizán, 1.

[...] Pardo, home de Johan Nunes, 2.

Affonsso [...], escudero, 2.

Affonsso Martin, clérigo de San Julián do Val, 2.

Afonso de Cordova, escribano, 1.

Afonso Eanes Iacob, notario publico del concejo de Pontevedra por la iglesia de Santiago, 1.

Afonso Eanes, clérigo, sobrino de Çibraao Peres, 1.

Afonso Lopes de Ball[...], arcediano del Salnés, 1.

Afonso Peres, escribano, 3.

Afonsso Gomes, escribano, 3.

Alvar Peres, hijo de Pedro Alvares de Soutomayor, 1.

Aras Fernandes de Meira, hijo de Fernan Anes/ Eanes de Meira y de dona Maria, 1, 3.

Aras Peres, notario, 2.

Bernald Eanes, notario de Pontevedra, 1.

Çibraao Peres, tío de Afonso Eanes, 1.

Domingo Eanes, clerigo, notario de Pontevedra y en el arcedianazgo de Salnés, 1.
Fernan [...], de San Cristóbal de Briallos, vicario en el arcedianato de Salnés, 1.

Fernán Anes Mourinno?, home de Johan Nunes, 2.

Fernan Anes/Eanes de Meira, marido de dona Maria, 1, 3.

Fernan Eanes de Meira, v. Fernan Anes/Eanes de Meira.

Fernan Goterrez de Salzeda, caballero, 1.

Fernan Soares/Suares, clérigo de San Julián do Val de Marín, 1, 2, 3.

Fernan Suares, v. Fernan Soares/Suares.

Fernan, carnicero, home de Johan Nunes, 2.

Fernando, don, rey de Castilla, [Fernando IV], 1.

Frei Afonso, abad de Armenteira, 3.

Frei Domingo, abad de Armenteira, 1.

Frei Johanne, fraile de Oseira, 1.

Frei/frey Afonso, celareiro de Armenteira, 1.

Frey Afonsso Domingues, dom, abad de Armenteira, 3.

Frey Domingo, abad de Armenteira, 3.

Frey Domingo, don, abad de Armenteira, 1.

Frey Fernan Martines, fraile de la Orden de San Francisco, 1.

Frey Fernando de Deça, fraile de Armenteira, 1. Frey Fernando, abad de Armenteira, 2. 
Frey Iohan, don, abad de Armenteira, 1.

Frey Johan, monje de Oseira, granjero de Marin, 3.

Frey Johanes, fraile de Oseira, 1.

Gomecius, arzobispo de Santiago de Compostela, 2.

Gomez, dom, arzobispo de Santiago, capellan mayor del rey y notario mayor del rey de Castilla y Leon, 2.

Gonçalvo Estevees, clerigo de San Julián do Val de Marín, 1.

Gonçalvo Martines?, chantre de Tuy y vicario general en el arcedianato del Salnés, 3.

Gonçalvo Peres Corvacho, chantre de Santiago, 3.

Gonçalvo Peres de Penente, home de Johan Nunes, 2.

Gonçalvo Peres, canónigo de Santiago, clérigo beneficiado de San Julián do Val, 2.

Ioan Nunes, 2.

Iohan do Rioo, 1.

Iohan Domingues, 1 .

Iohan Nunes, clerigo de Paradela, 3.

Iohan/Johan/Juan de Cordova, notario de Pontevedra, jurado en lugar de Bernald Eanes, 1 .

$\mathbf{J}_{\text {ohan de Cordova, v. Iohan/Johan/Juan de }}$ Cordova.
Johan Nunes, 2.

Johan Nunnez da Ponte, vecino de Pontevedra, juez de comisión por el arzobispo de Santiago, 2.

Johan Suares, 3.

Juan de Cordova, v. Iohan/Johan/Juan de Cordova.

Maria, dona, mujer de Fernan Anes/Eanes de Meira, 1, 3.

Martin Barregan, pescador, 3.

Martinno Anes, clérigo de coro, 3.

Martinno Anes/Martinno Eanes, clérigo de San Julián do Val de Marín, 3.

Martinno Eanes, clérigo, v. Martinno Anes.

Nuno Fernandes, cardenal, clérigo de San Julián do Val, 2.

Nuno Vidal, home de Johan Nunes, 2.

Pedro Alvares de Soutomayor, padre de Alvar Peres, 1.

Pedro Anes/Eanes, dito Salgeiro, de Vale, 1.

Pedro Eanes, dito Salgeiro, de Vale, v. Pedro Anes/Eanes.

Rui d'Ouvinna, escudero, 3.

Ruy Peres, notario de Pontevedra, 2.

Salgeiro, v. Pedro Anes/Eanes.

\section{ÍNDICE TOPONÍMICO}

\section{Advertencia al índice de topónimos}

Este índice está escrito en lengua gallega, pues el Nomenclátor de Galicia elaborado por los dictámenes de la Comisión de Toponimia y las normas dictadas por la Xunta de Galicia sólo contemplan como forma oficial la toponimia en dicha lengua.

Tanto los nombres oficiales actuales como sus variantes antiguas o no oficiales se ofrecen por orden alfabético.

El número o números que siguen a cada nombre indican el documento o documentos en el que se encuentra. 
Los nombres oficiales actuales van en versalita; si el nombre antiguo coincide con la denominación oficial actual, se remite a él. Así por ejemplo «Deça», que aparece en el doc. $\mathrm{n}^{\mathrm{o}} 1$, se remite a DEZA, nombre oficial actual de ese lugar. En el caso de topónimos homónimos, se escribe a continuación entre paréntesis su diferenciación: «Marin» remite a MARíN (vila), para no confundirlo con MARÍN (San Xián).

Los nombres que van en cursiva son topónimos localizados documentalmente, pero que han cambiado de denominación, como por ejemplo San Jullao do Val, hoy San Xián de Marín, del doc. $n^{\circ} 1$.

Las variantes antiguas $-\mathrm{y}$ las no oficiales hoy día-, se remiten siempre a su equivalente oficial actual, salvo las que no pudieron localizarse: así por ejemplo «Deça» se remite a DEZA.

Los apellidos de personas que son topónimos sólo son localizados e identificados en aquellos casos que hace precisa su ubicación: "Cordova» se remite a «CORDOBA». El ejemplo contrario puede darlo «Montillon» o «Salceda»: estos no se remiten a nada, dado que el topónimo «Montillón» y «Salceda» o sus posibles variantes está repetido en diferentes zonas geográficas, no pudiendose precisar a la que supuestamente pertenecen, ya que el documento donde aparece no ofrece ninguna información de ayuda al respecto.

Armenteira, a, Santa María de.- (moosteyro d'Armenteira, moosteiro/monasterio de Santa Maria d'Armenteira, Armenteyra, moesteiro/moesteyro d'Armenteyra, moesteyro de Santa Maria d'Armenteyra).Parroquia onde se encontra o mosteiro cisterciense do mesmo nome.- Prov. de Pontevedra. Concello de Meis.

Armenteira, monasterio de Santa Maria d', 1.V. ARMENTEIRA, A.

Armenteira, moosteyro d', 1.- v. ARMENTEIRA, A. Armenteira, moosteyro de Santa Maria d', 1.- v. ARMENTEIRA, A.

Armenteyra, 2.- v. ARMENTEIRA, A.

Armenteyra, moesteiro d', 2.- v. ARMENTEIRA, A. Armenteyra, moesteyro d', 2, 3.- v. ARMENTEIRA, A.

Armenteyra, moesteyro de Santa Maria d', 2, 3.v. ARMENTEIRA, A.
Briallos, [...] Christovoo de, 1.- v. BRiallos. BRiallos, San Cristovo de.- (Christovoo de Briallos).- Parroquia.- Prov. de Pontevedra. Concello de Portas.

Castela, 2.- v. castela.

CAStela.- (Castela).- Antigo reino hispánico.

CóRdobA.- (Cordova).- Provincia, concello e cidade española da rexión histórica de Andalucía. Prov. de Córdoba. Concello de Córdoba.

Cordova, Afonso de, 1.- v. CóRdoba.

Cordova, Iohan de, 1.- v. CÓRDOBA.

Cordova, Johan de, 1.- v. CÓRDOBA.

Cordova, Juan de, 1.- v. CóRdOBA.

Deça, Fernando de, 1.- v. DEZA.

DEZA, (Deça).- Comarca e antigua xurisdicción da Prov. de Pontevedra, situada entre o río Deza e o Arnego. 
Leom, 2.- v. LEÓN.

LEÓN.- (Leom).- Antigo reino hispánico; hoxe provincia, cidade, concello e rexión histórica de España. Prov. de León. Concello de León.

Logriçaa, Santo Andre de, 1.- v. LOURIZÁn. Lourizán, Santo André.- (Santo Andre de Logriçaa).- Parroquia.- Prov. de Pontevedra. Concello de Pontevedra.

Marin, 1, 3.- v. MARÍN (vila).

MARÍN, San Xián.- (San Juyao, iglesia de San Giao/Gyao do Val, San Jullao do Val, eglesia de San Juyaao do Val, eglesia/iglesia de Sam Giao do Vale, eglesia de San Juyao do Vale, Vale).- Parroquia.- Prov. de Pontevedra. Concello de Marín.

MARÍN.- (Marin).- Vila e concello.- Prov. de Pontevedra. Concello de Marín.

Meira, 1.- v. MEIRA.

Meira, Aras Fernandes de, 1.- v. MEIRA.

Meira, Fernan Anes/Eanes de, 1, 3.- v. MEIRA.

MEIRA.- (Meira, Meyra).- Concello, parroquia de Santa María e lugar.- Prov. de Lugo. Concello de Meira.

Meyra, 3.- v. MEIRA.

Montillon, [...] de, 1 .

Oseira, 1, 3.- v. oseira.

oseira, Santa María a Real de.- (Oseira).Mosteiro cisterciense, parroquia e lugar. Prov. de Ourense. Concello de San Cristovo de Cea.

Ouvinna, Rui d', 3.

Paradela, 3.

Penente, Gonçalvo Peres de, 2.- v. PEnEnTE. PENENTE, lugar de.- (Penente).- Parroquia de San Vicente de Nogueira. Prov. de Pontevedra. Concello de Meis.

Ponte, Johan Nunnez da, 2.

Pontevedra, 1, 2, 3.- v. PONTEVEDRA (cidade).

Pontevedra, moosteyro de San Françisco de, 1.v. PONTEVEDRA (mosteiro).

PONTEVEDRA, mosteiro ou convento de San Francisco de.- (moosteyro de San Françisco de).Convento franciscano do século XIII situado no centro da cidade, na praza da Ferre- ría.- Prov. de Pontevedra. Concello de Pontevedra.

Pontevedra, vila de, 1.- v. PONTEVEDRA (cidade). PONTEVEdRA.- (Pontevedra, vila de Pontevedra).-

Provincia, cidade e concello.- Prov. de Pontevedra. Concello de Pontevedra.

Rioo, Iohan de, 1.

Salnes, arçediagado/arçidiagado de, 1, 3.- v. SALNÉS, O.

SALNÉs, O.- (arçediagado/arçidiagado de Salnes).Antiga xurisdicción eclesiástica na prov. de Pontevedra. Hoxe é a comarca situada na costa da prov. de Pontevedra, que comprende, entre outros, os concellos de Sanxenxo, Meis, Poio, Cambados e O Grove, entre a ría de Pontevedra e a de Arousa.

Salzeda, Fernan Goterrez de, 1.

San Juyao, 1.- v. MARÍN (San Xian).

SANTIAGO DE COMPOSTEla, (Santiago, iglesia de Santiago).- Cidade, concello e arcebispado.Prov. de A Coruña. Concello de Santiago de Compostela.

Santiago, 2, 3.- v. SANTIAGO DE COMPOSTELA.

Santiago, iglesia de, 1.- v. SAntiago DE COMPOSTELA.

SOUTOMAIOR.- (Soutomayor).- Vila, concello e parroquia de San Salvador.- Prov. de Pontevedra. Concello de Soutomaior.

Soutomayor, Pedro Alvares de, 1.- v. SOUTOMAIOR.

Tui, 3.- v. TuI.

TUI.- (Tui).- Vila, concello e bispado.- Prov. de Pontevedra. Concello de Tui.

Val, San Giao do, iglesia de, 3.- Antiga denominación da actual parroquia de San Xián de MARÍN.

Val, San Gyao do, iglesia de, 3.- Antiga denominación da actual parroquia de San Xián de MARÍN.

Val, San Jullao do, 1.- Antiga denominación da actual parroquia de San Xián de MARíN.

Val, San Juyaao do, eglesia de, 1.- Antiga denominación da actual parroquia de San Xián de MARÍN.

Vale, 1.- Antiga denominación da actual parroquia de San Xián de MARíN. 
Vale, Sam Giao do, eglesia/iglesia de, 2.- Antiga denominación da actual parroquia de San Xián de MARíN.

Vale, San Juyao do, eglesia de, 1.- Antiga denominación da actual parroquia de San Xián de MARíN.

\section{BIBLIOGRAFÍA}

BOUZA-BREY, F., «Historia del Monasterio de Armenteira por fray Basilio Duarte (S. XVII)», Compostellanum, vol. VI, $\mathrm{n}^{\circ}$ 2, (abril-junio 1961), pp. 233-285.

Decreto 332/1996, do 26 de xullo, polo que se aproba o nomenclátor correspondente ás entidades de poboación da provincia de Ourense, Diario Oficial de Galicia, Santiago, 29 de Agosto de 1996.

Decreto 219/1998, do 2 de xullo, polo que se aproba o nomenclátor correspondente ás entidades de poboación da provincia de Pontevedra, Diario Oficial de Galicia, Santiago, 28 de Julio de 1998.

Decreto 6/2000, do 7 de xaneiro, polo que se aproba o nomenclátor correspondente ás entidades de poboación da provincia de Lugo, Diario Oficial de Galicia, Santiago, 25 de Enero de 2000.

Decreto 189/2003, do 6 de febreiro, polo que se aproba o nomenclátor correspondente ás entidades de poboación da Provincia da Coruña, Diario Oficial de Galicia, Santiago, 25 de marzo de 2003.

FRANCO ESPIÑO, B., «Notas sobre el origen del monasterio de Santa María de Armenteira», Estudios mindonienses, 17 (2001), pp. 575-586.

GRAN ENCICLOPEDIA GALlEGA, T. 2, voz «Armenteira, Santa María de», pp. 192-193.

LÓPEZ CARREIRA, A., Estudio sincrónico da economía de Ourense no século XV, Apéndice documental, Memoria de Licenciatura inédita, Universidad de Santiago.

LUCAS ÁLVAREZ, M., «Paleografía gallega. Estado de la cuestión», Anuario de estudios medievales, 21 (1991) pp. 419-470.

LUCAS ÁLVAREZ, M., «Para unas normas complementarias de transcripción de documentos en gallego», CEG, XII (1949), pp. 95-110.

MARÍN MARTÍNEZ, T. (dir.), Paleografia y Diplomática, 2 vols., Madrid, UNED, 1991. $5^{\text {a }}$ ed.

MIRALBES BEDERA, R; TORRES LUNA, Ma. P. de; RODRÍGUEZ MARTÍNEZ-CONDE, R., Mapa de límites de las parroquias de Galicia, Santiago, Universidad, 1979.

Nomenclátor de Galicia. [En línea]. Disponible en http.//xunta.es./nomenclator/index.htm (Consulta de fecha 7-diciembre-2003).

PÉREZ RODRÍGUEZ, F. J., «Benedictinos e cistercienses: un reconto dos mosteiros galegos a finais do século XII», Actas II Congreso Internacional sobre el Císter en Galicia y Portugal, Ourense, 1998, pp. 695-724. 
ROMANí MARTÍNEZ, M., Colección Diplomática do Mosteiro Cisterciense de Sta. María de Oseira (Ourense), 1025-1310, 2 vols., Santiago, Tórculo, 1989.

ROMANÍ MARTÍNEZ, M.; RODRÍGUEZ SUÁREZ, Ma. P., Libro Tumbo de Pergamino. Un códice medieval del monasterio de Oseira, Santiago, Tórculo, 2003.

VALlE PÉREZ, J. C., El Monasterio de Armenteira, Anejo de «Museo de Pontevedra», T. 31, 1977.

VÁZQUEZ ROUCO, S. San Xoán de Poio monasterio, coto, parroquia, Diputación Provincial de Pontevedra, Cambados, 1998.

YÁÑEZ NEIRA, D., El Monasterio de Armenteira y sus abades, Colección Museo de Pontevedra XXXIV, Diputación Provincial de Pontevedra, 1980. 